\section{Adrenalin - eine Dosis reicht nicht immer}

\author{
Mittel der Wahl bei einer anaphylaktischen Reaktion ist Adrenalin \\ intramuskulär, wobei manchmal mehr als eine Dosis gegeben wird. \\ Welche Umstände bei Kindern mehrfache Adrenalininjektionen \\ erforderlich machen, wurde in einer Fragebogenaktion ermittelt.
}

P ädiater der Mount Sinai School of Medicine, New York, verteilten Fragebögen an die Familien von insgesamt 542 Kindern und Jugendlichen bis zu 18 Jahren, die sich aufgrund einer durch Nahrungsmittel ausgelösten allergischen Reaktion vorstellten. Erhoben wurden u. a. demographische Daten, die Allergieanamnese sowie Einzelheiten zu Anaphylaxieepisoden, in denen Adrenalin injiziert wurde.

Von den Fragebögen konnten 413 ausgewertet werden. 78 Patienten (medianes Alter 4,5 Jahre) erlitten insgesamt 95 mit Adrenalin behandelte Reaktionen. Bei diesen 95 Reaktionen wurde in 77 Fällen $(81 \%)$ Adrenalin einmal appliziert, zwölf Reaktionen (13\%) machten eine zweimalige und sechs Reaktionen (6\%) eine dreimalige Injektion erforderlich. Häufigste Auslöser waren Erdnüsse, Baumnüsse und Kuhmilch. Patienten, die Mehrfachdosen benötigten, litten häufiger unter Asthma ( $p=0,027)$, die gegessene Menge an allergenem Nahrungsmittel hatte dagegen keinen Einfluss. In 94\% der Fälle wurde die zweite Adrenalindosis unter ärztlicher Aufsicht gegeben.

Die meisten Zweitinjektionen erfolgten innerhalb der ersten 30 Minuten nach Beginn der Symptome, was eher auf ein mangelndes Ansprechen auf die erste Dosis als auf einen möglichen biphasischen Verlauf der Reaktion als Grund für die Mehrfachapplikation hinweist. Neben krankheitsbedingten Faktoren könnte auch eine Unterdosierung, eine Überschreitung des Verfallsdatums oder ein unsachgemäßer Einsatz des Autoinjektors Grund für die erforderliche Zweitapplikation gewesen sein.

Fazit: In einem Kollektiv von Kindern und Jugendlichen, die eine durch Nahrungsmittel ausgelöste anaphylaktische Reaktion erlitten hatten, wurde in 19\% der Fälle mehr als eine Dosis Adrenalin intramuskulär injiziert. Möglicherweise ist Asthma ein Risikofaktor für die Notwendigkeit einer Mehrfachapplikation.

Järvinen KM et al. Use of multiple doses of epinephrine in food-induced anaphylaxis in children. J Allergy Clin Immunol 2008; 122: $133-8$

\section{Nahrungsmittel zu Hause aufdosieren}

\section{Kinder mit hohem Allergierisiko sollten bekannte Nahrungsmittel- allergene beim ersten Kontakt nur in langsam ansteigenden Mengen zu sich nehmen, um potenzielle klinische Reaktionen zu minimieren. Inwiefern die Eltern eine solche Aufdosierung zu Hause sicher durch- führen können, wollten niederländische Allergologen wissen.}

$\mathrm{K}^{\mathrm{i}}$ inder mit einem sehr hohen Allergierisiko sollten ihre ersten Mahlzeiten mit bekannten allergenen Nahrungsmitteln unter ärztlicher Aufsicht zu sich nehmen. Eine Alternative ist die Bereitstellung entsprechender Schemata für eine sichere Einführung dieser Nahrungsmittel im häuslichen Bereich. Zur Ermittlung von sicheren Vorgehensweisen wurden 63 Risikokinder in insgesamt 72 doppelblinden plazebokontrol-

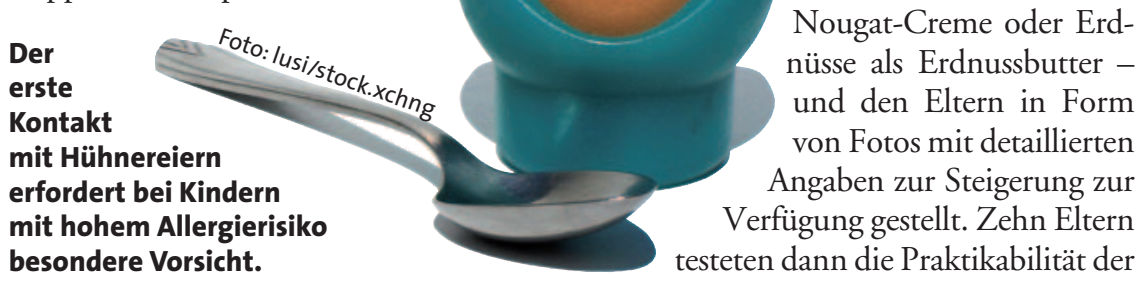

lierten Provokationstests gegenüber steigenden Mengen von Kuhmilch, Eiern, Erdnüssen, Soja, Haselnüssen und Walnüssen exponiert. Dabei wurden die initial sehr kleinen Mengen verdoppelt, größere Mengen um das Vier- bis Fünffache gesteigert.

Die so ermittelten Steigerungsstufen wurden in haushaltsübliche Kindermahlzeiten ,übersetzt“ z. B. Haselnüsse als NussNougat-Creme oder Erdnüsse als Erdnussbutter und den Eltern in Form von Fotos mit detaillierten Angaben zur Steigerung zu testeten dann die Praktikabilität de
Einführungspläne unter Alltagsbedingungen. Gleichzeitig wurden sie gefragt, in welchen Mengen sie ohne diese Informationen die Nahrungsmittel den Kindern gegeben hätten.

Die fotounterstützten Dosissteigerungspläne wurden gut angenommen. Es zeigte sich, dass bei Nutzung der Pläne den Kindern primär deutlich geringere Mengen allergener Nahrungsmittel angeboten wurden als ohne Anweisung. Die initial als sicher eingestuften Mengen waren tatsächlich sehr gering und umfassten zunächst nicht mehr als eine Messerspitze, einen Krümel oder einen Tropfen des Nahrungsmittels.

Fazit: Eltern, deren Kinder ein hohes familiäres Allergierisiko aufweisen, können bei diesen allergene Nahrungsmittel mit Hilfe fotografisch gestützer Schemata auch zu Hause vergleichsweise sicher einführen.

$b k$

Vlieg-Boerstra BJ et al. Ready-to-use introduction schedules for first exposure to allergenic foods in children at home. Allergy 2008; 63: 903-9 